\title{
Concurrent isolated retroperitoneal HGSC and STIC defined by somatic mutation analysis: a case report
}

\author{
Kazuaki Suda ${ }^{1}$, Hirofumi Nakaoka ${ }^{2,3}$, Chihiro Hata ${ }^{2,3}$, Natsumi Yahata ${ }^{4}$, Masanori Isobe ${ }^{1}$, Hitoshi Kameyama ${ }^{5}$, \\ Toshifumi Wakai ${ }^{5}$, Teiichi Motoyama ${ }^{6}$, Ituro Inoue ${ }^{2,3}$, Kosuke Yoshihara $^{1 *}$ (D) and Takayuki Enomoto ${ }^{1}$
}

\begin{abstract}
Background: Retroperitoneal high-grade serous carcinoma (HGSC) is extremely rare and the origin remains unclear. We present a case of retroperitoneal HGSC and coexisting serous tubal intraepithelial carcinoma (STIC), which is considered as the main origin of ovarian HGSC. We reviewed the available literature and discussed about the origin of this rare disease.

Case presentation: A 58-year-old female with a $93 \times 65 \times 62 \mathrm{~mm}$-solid tumor with a cystic part was located immediately dorsal to the rectum underwent bilateral salpingo-oophorectomy, total abdominal hysterectomy, and en bloc resection of the retroperitoneal tumor together with lower anterior resection of the rectum. Histological diagnosis was retroperitoneal HGSC and STIC at the right fallopian tube. Two deleterious somatic mutations in TP53 and BRCA2 genes were shared between retroperitoneal HGSC and STIC.
\end{abstract}

Conclusions: In addition to clinical features in the previous reports, our genetic findings suggest the origin of retroperitoneal HGSC might be STIC.

Keywords: Retroperitoneal high-grade serous carcinoma, Serous tubal intraepithelial carcinoma, Somatic mutation, Case report

\section{Background}

High-grade serous carcinoma (HGSC) is the most common histological type of ovarian cancer. Since genetic relationships between intraepithelial carcinoma of the fimbria and pelvic HGSC were clarified in 2007 [1, 2], a paradigm shift in the origin of ovarian cancer has been occurring. At present, serous tubal intraepithelial carcinoma (STIC) is recognized as the precursor lesion of HGSC [3-5], and a recent genomic study also endorses the theory that HGSC originates in fallopian tube [6]. In fact, the knowledge that STIC cells migrate onto the peritoneum corresponds to the clinical feature of HGSC characterized by peritoneal dissemination and massive ascites.

On the other hand, retroperitoneal HGSC is very rare case [7-13] and the precursor of retroperitoneal HGSC

\footnotetext{
* Correspondence: yoshikou@med.niigata-u.ac.jp

${ }^{1}$ Department of Obstetrics and Gynecology, Niigata University Graduate School of Medical and Dental Sciences, 1-757 Asahimachi-dori, Niigata 951-8510, Japan

Full list of author information is available at the end of the article
}

remains unclear. In this case report, we present a case of HGSC entirely existing in the retroperitoneal space without the evidence of lymphovascular invasion. Furthermore, we demonstrate that retroperitoneal HGSC and coexisting STIC shared the same somatic mutations using next-generation sequencing.

\section{Case presentation}

A 58-year-old (gravida 2, para 2) woman presented the nearby hospital complaining of persistent defecation disorder and vomiting. Although her family history was notable for pancreatic cancer in her father, there was no other familial history of cancer, including breast and ovarian cancer. Her past medical history is unremarkable. Her past surgical history includes right ovarian cystectomy for a dermoid cyst at the age of 30. A computed tomography (CT) scan showed a large pelvic tumor adjacent to the rectum. Laboratory findings showed that her serum level of cancer antigen (CA) 125 
increased to $315.2 \mathrm{IU} / \mathrm{ml}$. Magnetic resonance imaging (MRI) demonstrated that a $93 \times 65 \times 62 \mathrm{~mm}$-solid tumor with cystic parts was located immediately dorsal to the rectum (Fig. 1). CT and MRI showed no evidence of dissemination, lymph node metastasis, nor distant metastasis. Colonoscopy showed strong extrinsic compression at the rectum with intact mucosa; however, biopsy of the rectum and the tumor site was not performed during colonoscopy. Based on the MRI finding that a perirectal cystic tumor was present without peritoneal dissemination, stage IA ovarian cancer was suspected, and she was referred to our hospital for treatment. At laparotomy, the tumor was located dorsal to the rectum and existed entirely in the retroperitoneal space (Fig. 2a). There were no apparent lesions in the peritoneal cavity including bilateral adnexa, uterus, and peritoneum. Peritoneal washing cytology was negative. After bilateral salpingo-oophorectomy and total abdominal hysterectomy, en bloc resection of the retroperitoneal tumor together with lower anterior resection of the rectum was performed (Fig. 2b). Whereas the tumor was adhered to the rectal wall, the tumor itself was relatively well-capsulated and easily separated from surrounding fat tissues. Based on pathological diagnosis of the retroperitoneal tumor: high-grade serous carcinoma, she received 6 cycles of adjuvant chemotherapy with carboplatin, paclitaxel and bevacizumab according to the standard treatment strategy for ovarian cancer. After the combination therapy, bevacizumab was administered for 3 cycles of tri-weekly maintenance therapy but was discontinued because of general fatigue. She has been alive without evidence of recurrence for 20 months since her initial surgery.

\section{Pathological examination}

Macroscopically, the retroperitoneal tumor measured $80 \times 55 \times 35 \mathrm{~mm}$ in size and was divided into solid and cystic parts. The rectum and the peritoneum separated the tumor from the peritoneal cavity (Fig. 2c). The cyst part covered with a thick wall included bloody serous fluid. Removed genital organs (e.g. uterus, fallopian tubes, and ovaries) presented no abnormal gross findings except for uterine fibroids. Microscopically, a cyst wall which was composed of fibrous tissue contained hemosiderin-laden macrophages. A solid part of the tumor, characterized by extensive atypical nuclei and lace-like pattern by coalescence of papillae, revealed high-grade serous carcinoma (Fig. 3a). Although the tumor was invasive into rectal mascularis propria and adjacent fat tissues, the surgical margin, peritoneal invasion, and lymphovascular involvement were negative. In addition, neither cancer metastasis nor endosalpingiosis were identified in the lymph nodes in the adjacent fat tissue. There were no invasive lesions in genital organs but STIC lesion was detected at the right fallopian tube (Fig. 3d).

The cells of the retroperitoneal tumor and STIC were immunohistochemically positive for p53 (Fig. 3b \& e). Ki-67 was diffusely and partially positive in the tumor and STIC lesions, respectively (Fig. 3c and f).

\section{Target-gene sequencing of microdissected cancer cells from the retroperitoneal tumor}

The retroperitoneal tumor sample was immediately separated from surgical specimen, embedded in Tissue-Tek O.C.T. compound (Sakura Finetek) in a Tissue-Tek Cryomold (Sakura Finetek), and frozen in liquid nitrogen. Serial $8-\mu \mathrm{m}$-thick frozen sections were mounted on MMI Membrane Slides (Molecular Machines \& Industries), fixed with $100 \%$ methanol, and stained with toluidine blue. We performed laser-microdissection (LMD) using the MMI CellCut system (Molecular Machines \& Industries) to isolate tumor cells [14], followed by DNA extraction.

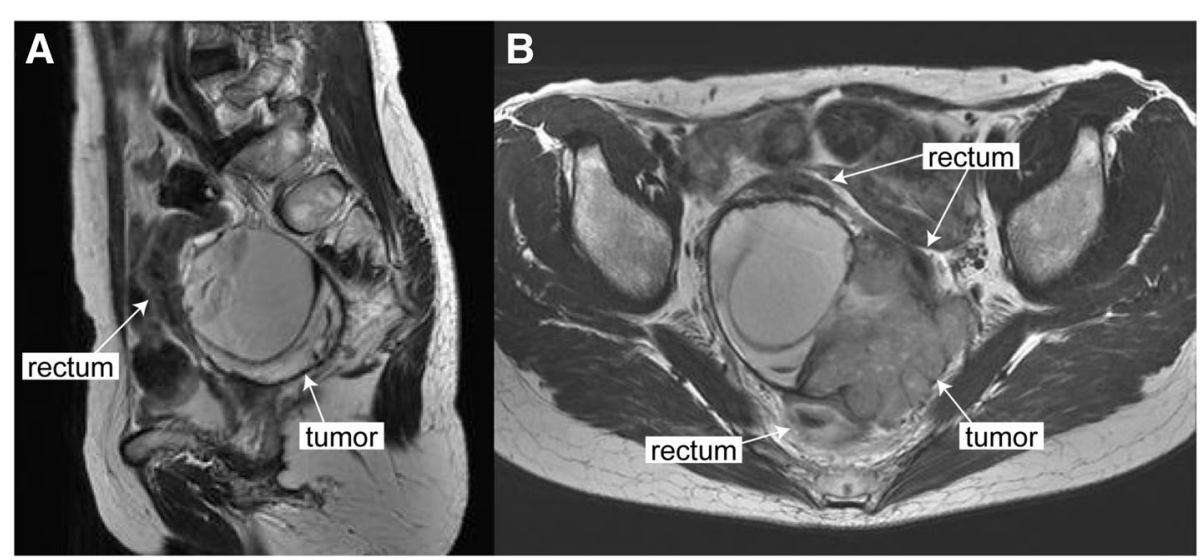

Fig. 1 Preoperative T2-weighted magnetic resonance imaging showed the pelvic mass composed of cystic and solid parts in $\mathbf{a}$ axial and $\mathbf{b}$ sagittal views. Arrowheads indicate the tumor and rectum in each panel 


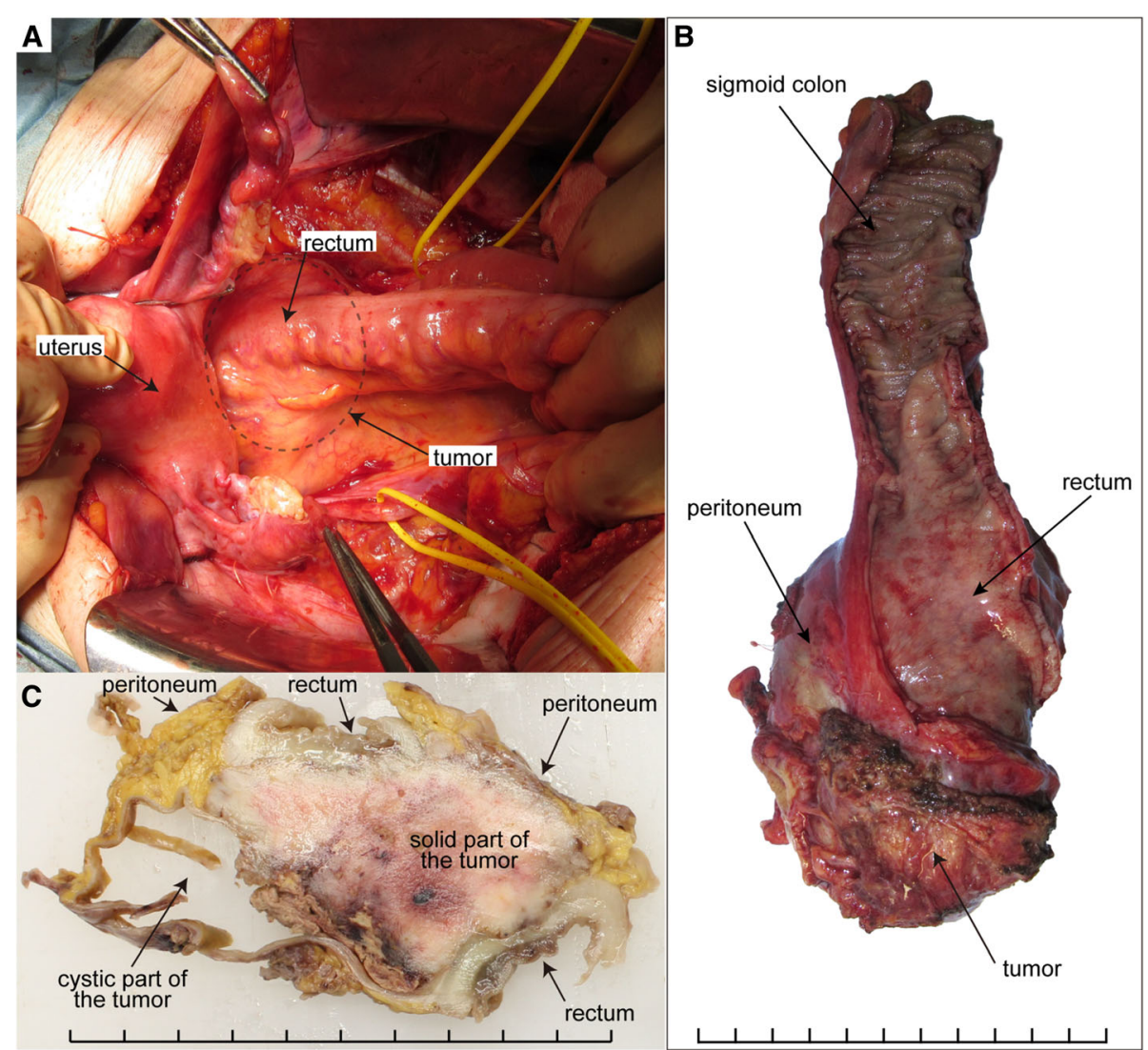

Fig. 2 a Pelvic laparotomy revealed the rectum compressed by the tumor in the mesorectum. The tumor was lying beneath the rectum at the area of dotted circle. Bilateral ureters were marked with yellow tapes. $\mathbf{b}$ The retroperitoneal capsuled tumor was resected with the rectum and the part of sigmoid colon. The tumor was surrounded by the rectum as a wrap. c A cross-sectional photograph of the surgical specimen. The tumor was completely separated from the peritoneal cavity with the rectum and peritoneum. Divisions of the scale bar in each panel show one $\mathrm{cm}$

Somatic mutations were investigated by target gene sequencing in the retroperitoneal tumor. We selected 120 genes that were frequently mutated in ovarian and endometrial cancers from the TumorPortal website [14, 15] and involved in the homologous recombination repair pathway. We performed target sequencing of the 120 genes with a pool and capture method described in our previous study $[14,16]$. The DNA libraries from the retroperitoneal tumor and the peripheral blood samples were sequenced via Illumina HiSeq 2500 platform in a rapid run mode with a $2 \times 100$ bp paired-end module. Referring to sequencing data from peripheral blood as a control, we called somatic mutations using Strelka software [17].

Somatic mutations detected in retroperitoneal tumor were listed in Table 1. Among six non-silent somatic mutations, a mutation of TP53 (c.536A > G, p.179H > R) showed the highest mutant allele frequency (MAF) of 0.94. A nonsense mutation of BRCA2 (c.6385G > T, p.2129E $>$ X) also showed high MAF of 0.84 . The MAFs of these two mutations were significantly higher than 0.5 (binomial test, $P<0.0001$ ), suggesting the presence of either loss-of-heterozygosity events or homozygous mutations at these sites. We detected no obviously pathogenic mutations in the patient's germline data obtained by sequencing for her blood cells.

\section{Deep sequencing of variant sites}

To explore the genetic relevance between the retroperitoneal tumor and STIC lesion, deep sequencing was performed focusing on the two variant sites (TP53: c.536A and BRCA2: c.6385G) which were the most dominant in the retroperitoneal tumor. To obtain the STIC lesion, LMD was also performed using formalin-fixed paraffin-embedded (FFPE) tissue sections of $10-\mu \mathrm{m}$ thickness. For LMD experiment, one per ten serial slides ( $n=1, n=11$, etc.) was immunostained for P53. DNA samples derived from the retroperitoneal tumor and STIC were amplified by PCR reactions using the following oligonucleotide primers. Forward and reverse primers for TP53 c.536A $>\mathrm{G} \quad[\mathrm{p} .179 \mathrm{H}>\mathrm{R}]$ were 5'-CTGCTCACCATCGCTATCTG-3' and 5'-CACA TGACGGAGGTTGTGAG-3', respectively. For BRCA2 c.6385G > T [p.2129E > X], forward and reverse primers 


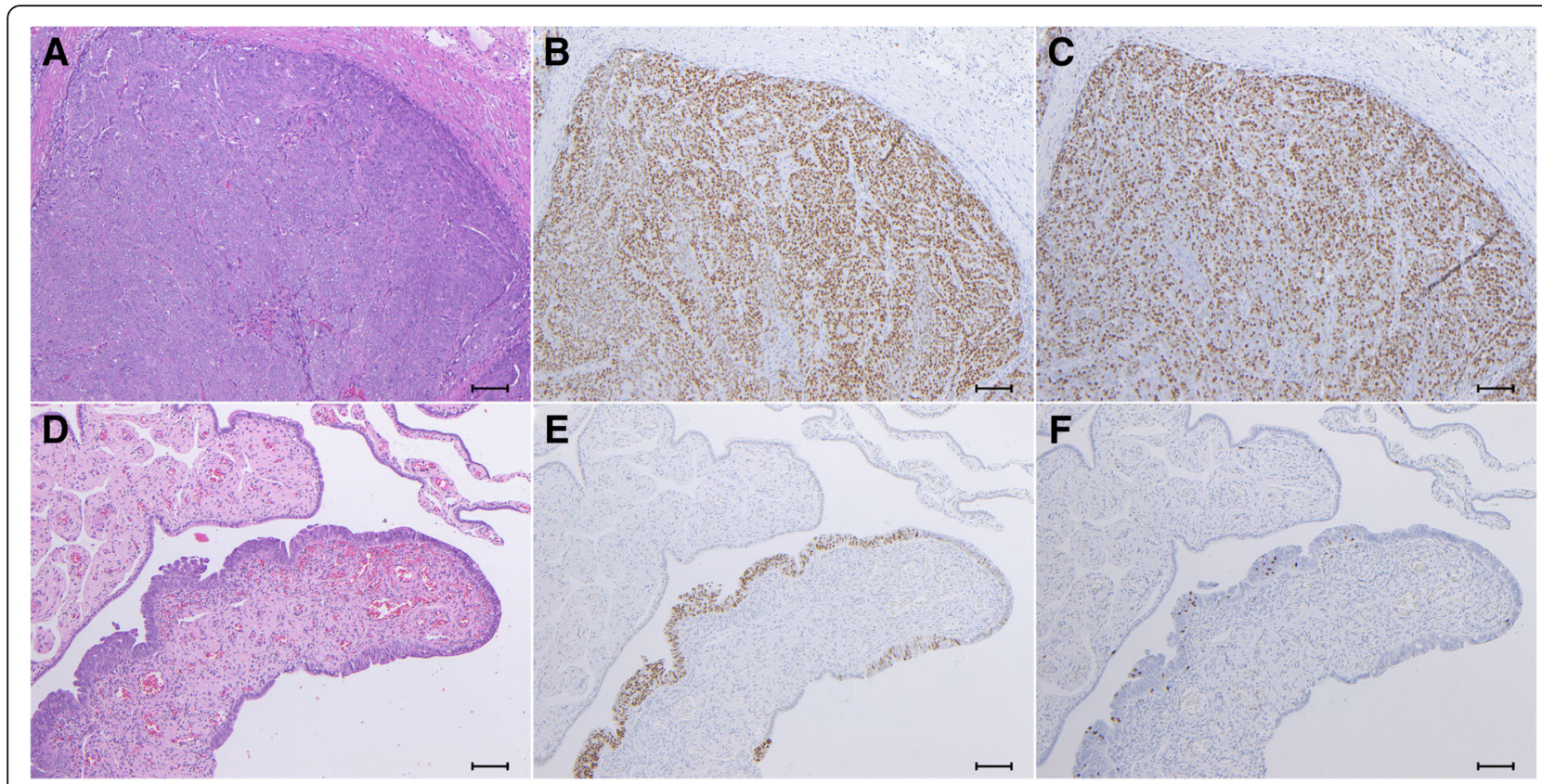

Fig. 3 Histological images of $\mathbf{a}, \mathbf{b}, \mathbf{c}$ the retroperitoneal HGSC and $\mathbf{d}, \mathbf{e}, \mathbf{f}$ STIC examined by $\mathbf{a}$, $\mathbf{d}$ hematoxylin and eosin staining, immunohistochemical staining for $\mathbf{b}$, e p53 and $\mathbf{c}, \mathbf{f}$ Ki-67. All panels are shown at power field of $\times 100$. Scale bars show $100 \mu \mathrm{m}$

were 5' ${ }^{\prime}$ CTGCTCACCATCGCTATCTG-3' and 5'-CACATGACGGAGGTTGTGAG-3', respectively. The PCR products were subjected to a deep sequencing via Illumina MiSeq v2 platform with a $2 \times 150 \mathrm{bp}$ paired-end module. The tag counts of paired-end sequences supporting the reference and mutant alleles were measured by using only high confidence base calls (base quality $>20$ ) at the mutation sites.

As a result, the somatic mutations of TP53 and $B R C A 2$ were shared between the retroperitoneal tumor and STIC lesion (Table 2). The MAFs of these two mutations were lower in STIC lesion. There is a possibility that STIC lesion was inadequately isolated from surrounding normal tubal epithelium, stroma and infiltrating immune cells through LMD, because the area of STIC lesion was limited.

\section{Discussion}

In this report, we presented HGSC existing entirely in retroperitoneal space without intraperitoneal malignant lesion except STIC in the fallopian tube, and demonstrated the genetic relationship between retroperitoneal HGSC and STIC lesion. In addition, extremely high MAF of the two mutations suggested that mutations might occur at the early stage of tumorigenesis, and it seemed reasonable to interpret that the origin of the retroperitoneal HGSC might be STIC.

To date, seven case reports of retroperitoneal HGSC are retrieved by searching NCBI PubMed service with the keywords of "retroperitoneal serous carcinoma" and "serous carcinoma \& retroperitoneum" (Table 3) [7-13]. All patients were female, and onset age ranged from 11 to 75 . In these reports, locations of the tumors varied from pelvis to upper abdomen in the retroperitoneal space. To explain how HGSC arose in retroperitoneal space, some theories represented by metaplasia of coelomic mesothelium and supernumerary ovary were discussed [7-12]. Unfortunately, no detailed analysis was performed in these reports to identify the pathogenesis of retroperitoneal HGSC and the origin of

Table 1 Mutations detected by target-gene sequencing of the retroperitoneal high-grade serous carcinoma

\begin{tabular}{lllll}
\hline Gene & Amino acid (DNA) substitution & No. wild-type reads & No. mutant reads & MAF \\
\hline TP53 & $179 \mathrm{H}>\mathrm{R}(536 \mathrm{~A}>\mathrm{G})$ & 3 & 50 & 89 \\
BRCA2 & $2129 \mathrm{E}>$ X $(6385 \mathrm{G}>\mathrm{T})$ & 17 & 39 & 0.94 \\
MLL4 & $2009 \mathrm{~A}>\mathrm{V}(6026 \mathrm{C}>\mathrm{T})$ & 21 & 33 & 0.65 \\
SIGLEC9 & $\mathrm{Y} 117 \mathrm{H}(349 \mathrm{~T}>\mathrm{C})$ & 33 & 15 & 0.50 \\
MUC6 & $1758 \mathrm{H}>\mathrm{L}(5273 \mathrm{~A}>\mathrm{T})$ & 179 & 0.08 \\
\hline
\end{tabular}


Table 2 Deep sequencing information of the tumor and STIC

\begin{tabular}{llllll}
\hline Targeted gene & Amino acid (DNA) substitution & Sample & No. wild-type reads & No. mutant reads & MAF \\
\hline TP53 & $179 \mathrm{H}>\mathrm{R}(536 \mathrm{~A}>\mathrm{G})$ & Tumor & 1685 & 7383 & 110,704 \\
& & STIC & 152,901 & 0.814 \\
BRCA2 & 2129E $>$ X (6385G $>$ T) & Tumor & 6162 & 39,716 & 0.420 \\
& & STIC & 96,243 & 86,018 & 0.472 \\
\hline
\end{tabular}

MAF denotes mutant allele frequency

retroperitoneal HGSC remains unclear. In the two reports, bilateral salpingooophorectomy (BSO) was performed but there was no description about coexisting STIC. As shown in the article focused on the pathogenesis of epithelial ovarian cancer [18], a small STIC is frequently missed. In this case, STIC was diagnosed by a skilled gynecological pathologist (TM) based on morphological appearance and immunostaining for p53. For more accurate diagnosis of STIC, p16 was shown to be a useful biomarker in identifying STIC, separate from morphologically normal fallopian tube epithelium or HGSC [19, 20]. Based on the results in earlier studies, we attempted immunostaining for p16. However, all sections having the STIC lesion were used for performing LMD, which eliminated the opportunity of immunostaining for $\mathrm{p} 16$ or any further examination.

There are some theories about how STIC cells were transferred into the retroperitoneal space without peritoneal spread. Firstly, STIC cells could have migrated by lymphovascular metastasis in spite of "intraepithelial carcinoma" as Schneider et al. have described. They claimed that STIC should be regarded as a malignant lesion with metastatic potential by presenting that STIC cases accompanied lymph node metastases without any other intra-abdominal/peritoneal spread [21]. In this case, there were no evidence of the lympovascular invasion pathologically in surgical specimens including fallopian tubes. The patient was incompletely staged for peritoneal disease (or ovarian cancer), lacking pelvic and para-aortic lymphadenectomy, omentectomy, and peritoneal biopsy. Some articles have demonstrated microscopic metastasis at components for FIGO staging in early stage ovarian cancer. In the study of occult metastasis, lymphatic and omental/peritoneal involvement of serous carcinoma was detected at the rate of $17 \%(12 /$ $69)$ and $4 \%(3 / 69)$, respectively [22]. Another study reported that microscopic metastasis was found at pelvic peritoneum or omentum in two (9\%) of 23 patients with apparent early serous ovarian cancer [23]. However, in apparent early stage epithelial ovarian cancer, random peritoneal biopsy and omentectomy beyond careful inspection of peritoneum was considered to have little significance [24]. However, we could not further assess the metastasis of STIC cells.

Secondly, tubal epithelial cells could have implanted directly into the mesentery, which resulted in endosalpingiosis. Kurman et al. have described the association between proliferative tubal epithelium and low-grade serous tumor and proposed the possibility that endosalpingiosis results from implantation of tubal epithelial cells [25]. Besides, it was reported that endosalpingiosis could be the origin of serous carcinoma in the mesentrium. McCoubrey et al. pathologically demonstrated the transformation from benign ciliated serous-type epithelium to well-differentiated serous carcinoma in a cystic endosalpingiosis in the mesosigmoid [26]. Combining these studies, we reasoned that TP53-mutated tubal epithelial cells developed into HGSC in the mesocolon via endosalpingiosis. In addition, our inference could be theoretically supported by the concept of "precursor escape," which was recently raised by Crum [27]. Based on this novel concept, TP53-mutated tubal epithelial cells already have the potential to be the precursor of HGSC at other than tubal epithelium since they were p53

Table 3 Previous case reports of retroperitoneal HGSC

\begin{tabular}{|c|c|c|c|c|c|}
\hline Author (year) & Age & Sex & Tumor size $(\mathrm{cm})$ & Location of the retroperitoneal tumor & Prognosis \\
\hline Ulbright (1983) [7] & 11 & female & $18 \times 13 \times 11$ & Adherent to posterior pubic symphysis, involving the right retroperitoneum & N/A \\
\hline Caruncho (1993) [8] & 49 & female & $9 \times 6 \times 5$ & From the left ureteropyelic junction to the upper limit of the previs & N/A \\
\hline Kurosaki (1998) [9] & 38 & female & 6 & Adherent to the lower pole of the right kidney & $24 \mathrm{M}$ alive \\
\hline Kaku (2004) [10] & 44 & female & $6 \times 3.5 \times 3$ & Surrounded by the left kidney, the aorta and the psoas major muscle & $23 \mathrm{M}$ alive \\
\hline Demir (2007) [11] & 40 & female & $15 \times 13 \times 10$ & In the right suprarenal fossa & N/A \\
\hline lura (2009) [12] & 66 & female & $20 \times 9.5 \times 8.5$ & Adjacent to ascending colon, from lower limit of the liver to the ileocecum & $32 \mathrm{M}$ alive \\
\hline Arichi (2011) [13] & 75 & female & $4.8 \times 5 \times 5$ & Attached to the right kidney and the liver & $6 \mathrm{M}$ alive \\
\hline Present case & 58 & female & $8 \times 5.5 \times 3.5$ & In the mesorectum & $16 \mathrm{M}$ alive \\
\hline
\end{tabular}

N/A denotes not available 
signature or early serous proliferations. In this case, one possibility is that p53 signature might have migrated into the mesentery and resulted in HGSC, while p53 signature in the fallopian tube grew into STIC.

\section{Conclusions}

We presented a case of retroperitoneal HGSC possessing genetic relationship with STIC, suggesting that STIC is the precursor of retroperitoneal HGSC. When the tumor lying in the retroperitoneal space was diagnosed with HGSC pathologically, complete resection of the tumor with BSO and elaborated pathological examination of fallopian tubes should be recommended.

\section{Abbreviations \\ BSO: Bilateral salpingo-oophorectomy; CA125: Cancer antigen 125; CT: Computed tomography; HGSC: High-grade serous carcinoma; MRI: Magnetic resonance imaging; PCR: Polymerase Chain Reaction; STIC: Serous tubal intraepithelial carcinoma}

\section{Acknowledgements}

We are grateful to Junko Kajiwara, Junko Kitayama, Yumiko Sato, and Anna Ishida for their technical assistance.

\section{Funding}

This work was supported in part by Japan Society for the Promotion of Science (JSPS) JP16H06267 (Grant-in-Aid for Young Scientists A for K. Yoshihara).

\section{Availability of data and materials}

The datasets used and/or analyzed during the current study are available from the corresponding author on reasonable request.

\section{Authors' contribution}

$\mathrm{KS}$ and $\mathrm{HN}$ performed and analyzed experiments. KS and KY interpreted all data, prepared the figures, and drafted the manuscript. All authors read and approved the final manuscript.

\section{Ethics approval and consent to participate}

Institutional ethics review board at Niigata University approved this study. The patient gave written informed consent.

\section{Consent for publication}

Written informed consent was obtained from the patient

\section{Competing interests}

The authors declare that they have no competing interests.

\section{Publisher's Note}

Springer Nature remains neutral with regard to jurisdictional claims in published maps and institutional affiliations.

\footnotetext{
Author details

'Department of Obstetrics and Gynecology, Niigata University Graduate School of Medical and Dental Sciences, 1-757 Asahimachi-dori, Niigata 951-8510, Japan. ${ }^{2}$ Department of Genetics, School of Life Sciences, Graduate University for Advanced Studies (SOKENDAI), Hayama, Japan. ${ }^{3}$ Division of Human Genetics, National Institute of Genetics, Mishima, Japan. ${ }^{4}$ Department of Obstetrics and Gynecology, Niigata Prefectural Shibata Hospital, Shibata, Japan. ${ }^{5}$ Division of Digestive and General Surgery, Niigata University Graduate School of Medical and Dental Sciences, Niigata, Japan. ${ }^{6}$ Department of Molecular and Diagnostic Pathology, Niigata University Graduate School of Medical and Dental Sciences, Niigata, Japan.
}

Received: 26 November 2018 Accepted: 1 February 2019

Published online: 11 February 2019

\section{References}

1. Lee Y, Miron A, Drapkin R, Nucci MR, Medeiros F, Saleemuddin A, Garber J, Birch C, Mou H, Gordon RW, et al. A candidate precursor to serous carcinoma that originates in the distal fallopian tube. J Pathol. 2007;211(1): 26-35.

2. Kindelberger DW, Lee Y, Miron A, Hirsch MS, Feltmate C, Medeiros F, Callahan MJ, Garner EO, Gordon RW, Birch C, et al. Intraepithelial carcinoma of the fimbria and pelvic serous carcinoma: evidence for a causal relationship. Am J Surg Pathol. 2007;31(2):161-9.

3. Kuhn E, Kurman RJ, Vang R, Sehdev AS, Han G, Soslow R, Wang TL, Shih le M. TP53 mutations in serous tubal intraepithelial carcinoma and concurrent pelvic high-grade serous carcinoma--evidence supporting the clonal relationship of the two lesions. J Pathol. 2012;226(3):421-6.

4. McDaniel AS, Stall JN, Hovelson DH, Cani AK, Liu CJ, Tomlins SA, Cho KR. Next-generation sequencing of tubal intraepithelial carcinomas. JAMA Oncol. 2015;1(8):1128-32.

5. Bashashati A, Ha G, Tone A, Ding J, Prentice LM, Roth A, Rosner J, Shumansky K, Kalloger S, Senz J, et al. Distinct evolutionary trajectories of primary high-grade serous ovarian cancers revealed through spatial mutational profiling. J Pathol. 2013;231(1):21-34.

6. Labidi-Galy SI, Papp E, Hallberg D, Niknafs N, Adleff V, Noe M, Bhattacharya $\mathrm{R}$, Novak M, Jones S, Phallen J, et al. High grade serous ovarian carcinomas originate in the fallopian tube. Nat Commun. 2017:8(1):1093.

7. Ulbright TM, Morley DJ, Roth LM, Berkow RL. Papillary serous carcinoma of the retroperitoneum. Am J Clin Pathol. 1983;79(5):633-7.

8. Caruncho M, Pombo F, Arnal-Monreal F. Primary retroperitoneal serous cystadenocarcinoma of 'ovarian-type': US and CT findings. Eur J Radiol. 1993:17(2):115-6.

9. Kurosaki Y, Kuramoto K. Case report: serous cystadenocarcinoma of the retroperitoneum: CT and sonographic appearance. Clin Radiol. 1998;53(12): 916-8.

10. Kaku M, Ohara N, Seima Y, Imanishi K, Tomura N, Kobayashi A, Yamasaki M, Hirata $Y$, Murao S. A primary retroperitoneal serous cystadenocarcinoma with clinically aggressive behavior. Arch Gynecol Obstet. 2004;270(4):302-6.

11. Demir MK, Unlu E, Genchellac H, Temizoz O, Ozdemir H: Primary serous papillary carcinoma of the retroperitoneum: magnetic resonance imaging findings with pathologic correlation. Australas Radiol 2007, 51 Spec No.: B71-B73.

12. Iura A, Sasajima Y, Katsumata N, Kasamatsu T. Serous adenocarcinoma of the retroperitoneum, as a type of multifocal mullerian carcinoma. Int J Clin Oncol. 2009:14(3):254-7.

13. Arichi N, Yasumoto H, Mitsui Y, Hiraoka T, Honda S, Shiina H, Igawa M. A case of primary retroperitoneal serous adenocarcinoma. Int J Urol. 2011; 18(12):844-6.

14. Suda K, Nakaoka H, Yoshihara K, Ishiguro T, Tamura R, Mori Y, Yamawaki K, Adachi S, Takahashi T, Kase H, et al. Clonal expansion and diversification of Cancer-associated mutations in endometriosis and Normal endometrium. Cell Rep. 2018:24(7):1777-89.

15. Lawrence MS, Stojanov P, Mermel CH, Robinson JT, Garraway LA, Golub TR, Meyerson M, Gabriel SB, Lander ES, Getz G. Discovery and saturation analysis of cancer genes across 21 tumour types. Nature. 2014;505(7484): 495-501.

16. Ahmadloo S, Nakaoka H, Hayano T, Hosomichi K, You H, Utsuno E, Sangai T, Nishimura M, Matsushita K, Hata A, et al. Rapid and cost-effective highthroughput sequencing for identification of germline mutations of BRCA1 and BRCA2.J Hum Genet. 2017:62(5):561-7.

17. Saunders CT, Wong WS, Swamy S, Becq J, Murray LJ, Cheetham RK. Strelka: accurate somatic small-variant calling from sequenced tumor-normal sample pairs. Bioinformatics (Oxford England). 2012;28(14):1811-7.

18. Kurman RJ, Shih le M. The origin and pathogenesis of epithelial ovarian cancer: a proposed unifying theory. Am J Surg Pathol. 2010;34(3):433-43.

19. Novak M, Lester J, Karst AM, Parkash V, Hirsch MS, Crum CP, Karlan BY, Drapkin R. Stathmin 1 and p16(INK4A) are sensitive adjunct biomarkers for serous tubal intraepithelial carcinoma. Gynecol Oncol. 2015;139(1):104-11.

20. Sehdev AS, Kurman RJ, Kuhn E, Shih le M. Serous tubal intraepithelial carcinoma upregulates markers associated with high-grade serous carcinomas including Rsf-1 (HBXAP), cyclin E and fatty acid synthase. Mod Pathol. 2010;23(6):844-55. 
21. Schneider S, Heikaus S, Harter P, Heitz F, Grimm C, Ataseven B, Prader S, Kurzeder C, Ebel T, Traut A, et al. Serous tubal intraepithelial carcinoma associated with Extraovarian metastases. International journal of gynecological cancer : official journal of the International Gynecological Cancer Society. 2017;27(3):444-51.

22. Ayhan A, Gultekin M, Celik NY, Dursun P, Taskiran C, Aksan G, Yuce K: Occult metastasis in early ovarian cancers: risk factors and associated prognosis. Am J Obstet Gynecol 2007, 196(1):81.e81-86.

23. Shroff R, Brooks RA, Zighelboim I, Powell MA, Thaker PH, Mutch DG, Massad LS. The utility of peritoneal biopsy and omentectomy in the upstaging of apparent early ovarian cancer. International journal of gynecological cancer : official journal of the International Gynecological Cancer Society. 2011; 21(7):1208-12.

24. Powless CA, Bakkum-Gamez JN, Aletti GD, Cliby WA. Random peritoneal biopsies have limited value in staging of apparent early stage epithelial ovarian cancer after thorough exploration. Gynecol Oncol. 2009;115(1):86-9.

25. Kurman RJ, Vang R, Junge J, Hannibal CG, Kjaer SK, Shih le M. Papillary tubal hyperplasia: the putative precursor of ovarian atypical proliferative (borderline) serous tumors, noninvasive implants, and endosalpingiosis. Am J Surg Pathol. 2011;35(11):1605-14.

26. McCoubrey A, Houghton O, McCallion K, McCluggage WG. Serous adenocarcinoma of the sigmoid mesentery arising in cystic endosalpingiosis. J Clin Pathol. 2005;58(11):1221-3.

27. Soong TR, Howitt BE, Horowitz N, Nucci MR, Crum CP. The fallopian tube, "precursor escape" and narrowing the knowledge gap to the origins of high-grade serous carcinoma. Gynecol Oncol. 2018

Ready to submit your research? Choose BMC and benefit from:

- fast, convenient online submission

- thorough peer review by experienced researchers in your field

- rapid publication on acceptance

- support for research data, including large and complex data types

- gold Open Access which fosters wider collaboration and increased citations

- maximum visibility for your research: over $100 \mathrm{M}$ website views per year

At $\mathrm{BMC}$, research is always in progress.

Learn more biomedcentral.com/submissions 